\title{
Jugend und Politik
}

\section{Heinz-Hermann Krüger • Thomas Rauschenbach}

Die Frage nach der politischen Integrationsbereitschaft der Jugend bzw. nach dem Verhältnis von Jugendlichen zu Politik und Demokratie gehört zu den zentralen Themen der Jugendforschung in Deutschland, die seit der Nachkriegszeit kontinuierlich untersucht worden ist. Diese Forschungstradition reicht von Schelskys Standardwerk zur skeptischen Generation aus dem Jahr 1961 (Schelsky 1961), in dem dieser unter Bezug auf die Daten der EMNID-Studien aus den 1950er-Jahren das Bild einer politikdistanzierten Nachkriegsgeneration entworfen hat, bis hin etwa zu der aktuellen Shell-Studie aus dem Jahr 2019 (Shell Holding Deutschland 2019), die das Porträt einer gegenwärtigen Jugendgeneration zeichnet, deren politische Orientierungen im Spektrum zwischen weltoffenen und kosmopolitischen Einstellungen auf der einen und nationalpopulistischen Haltungen auf der anderen Seite verortet werden.

Die Intensität der empirischen Erforschung des Spannungsverhältnisses zwischen Jugend und Politik wurde in den letzten Jahrzehnten dabei jedoch jeweils stark von gesamtgesellschaftlichen Rahmenbedingungen sowie dem Auftauchen unterschiedlicher sozialer Protestbewegungen mit beeinflusst. So setzte z. B. im Gefolge des Zusammenbruchs der DDR, der schnellen Wiedervereinigung beider deutscher Staaten sowie des Erstarkens rechtsextremer Gruppierungen seit Beginn der 1990er-Jahre ein Boom von quantitativen Studien in Gestalt von einmaligen Querschnittsbefragungen, Zeitwandelstudien oder wenigen Längsschnittstudien, aber auch qualitativbiografischen oder gruppenbezogenen Studien ein, die die Wertvorstellungen und

Prof. Dr. H.-H. Krüger $(\bowtie)$

Erziehungswissenschaften - Institut für Pädagogik, Martin-Luther-Universität Halle-Wittenberg,

Haus 4, Raum 9, 06099 Halle (Saale), Deutschland

E-Mail: heinz-hermann.krueger@paedagogik.uni-halle.de

Prof. Dr. T. Rauschenbach

Deutsches Jugendinstitut, Nockherstr. 2, 81541 München, Deutschland

E-Mail: rauschenbach@dji.de 
politischen, auch rechtsextremen, Orientierungen von Jugendlichen insbesondere im Ost-West-Vergleich untersucht haben (im Überblick Krüger und Grunert 2020).

Nach dieser Hochphase am Ausgang des 20. Jahrhunderts haben Studien, die sich mit dem Themenfeld Jugend und Politik beschäftigen, in den vergangenen zwei Jahrzehnten jedoch an Bedeutung verloren. Dies dokumentiert sich u. a. auch in dem Tatbestand, dass sich Wissenschaftlerinnen und Wissenschaftler aus Deutschland im Unterschied zu den Vorgängerstudien an der dritten internationalen Civic Education Study im Jahr 2009 nicht beteiligt haben (Abs et al. 2017, S. 14).

Erst in jüngster Zeit sind wieder verstärkt einige empirische Studien durchgeführt worden, die an klassische Themen der politischen Sozialisationsforschung wie politische Involviertheit, politisches Wissen oder zivilgesellschaftliches Engagement anknüpfen und diese Phänomene unter veränderten theoretischen und methodischen Perspektiven in den Blick nehmen oder die vor dem Hintergrund des Anstieges rechtpopulistischer Orientierungen in Deutschland und Europa sowie damit einhergehender politischer und gesellschaftlicher Polarisierungsprozesse die Auswirkungen dieser Entwicklungen auf die politische Orientierungen von Jugendlichen untersuchen.

Aus dem Spektrum dieser aktuellen Untersuchungen werden in diesem Heft die Ergebnisse von vier Studien vorgestellt, die sich mit dem Wandel des politischen Interesses und der Engagementbereitschaft von Jugendlichen und jungen Erwachsenen in einer Längsschnittperspektive, mit dem Verhältnis von schulischen Lernbedingungen, politischem Wissen und Einstellungen von Schülerinnen und Schülern zu Europa, mit der Verbreitung menschenfeindlicher Einstellungen bei Jugendlichen auch im Vergleich zu anderen Bevölkerungsgruppen sowie mit dem Umgang von Jugendlichen mit dem Protestphänomen „PEGIDA“ beschäftigt haben. Ergänzt werden diese durch einen internationalen Beitrag, der den Blick vor allem auf die unterschiedlichen Facetten und die Bedingungskontexte politischer Partizipationsformen bei Jugendlichen in 14 europäischen Ländern richtet.

\section{Einführung in die einzelnen Beiträge}

Martina Gille und Susanne Kuger versuchen in ihrem Artikel vor allem zwei Fragen zu beantworten. Zum einen wird untersucht, inwiefern politische Involviertheit und zivilgesellschaftliches Engagement stabile Merkmale einer Person im Verlaufe des Jugend- und frühen Erwachsenenalters sind. Zum anderen wird analysiert, in welchem Ausmaß sich politisches Interesse und ehrenamtliches Engagement während der Jugendalters und des jungen Erwachsenenalters gegenseitig beeinflussen. Nach einem Überblick zu dem überschaubaren Forschungsstand zu diesen Themenfeldern vor allem unter einer längsschnittlichen Perspektive stellen die Autorinnen das methodische Design ihrer quantitativen Längsschnittstudie vor. Ihre Auswertungen stützen sich auf den Datensatz der AIDA-Studien des DJI, die in den Jahren 2009, 2014 und 2018 bei der gleichen Personengruppe durchgeführt worden sind. Die komplexen längsschnittlichen Analysen bestätigen die Hypothese, dass das Interesse an Politik und die subjektive Bedeutung des Lebensbereichs Politik im Verlaufe der Jugendphase und nach dem Übergang ins frühe Erwachsenenalter zunimmt. 
Das ehrenamtliche Engagement geht hingegen mit zunehmenden Alter, insbesondere nach dem Eintritt in den Beruf, zurück. Zudem wird aufgezeigt, dass sich der theoretisch unterstellte Zusammenhang zwischen dem politischen Interesse und dem ehrenamtlichen Engagement erst im jungen Erwachsenenalter deutlich nachweisen lässt.

In dem Beitrag von Katrin Hahn-Lautenberg und Hermann Josef Abs wird das Verhältnis von schulischen Bedingungen und politischem Wissen zu Einstellungen gegenüber Supranationalität, überstaatlichem Institutionenvertrauen und zur Performanz der Europäischen Union bei 14-jährigen Schülerinnen und Schülern analysiert. Ausgehend von Konzepten der politischen Kulturforschung, insbesondere Theorien der sozialen Unterstützung, werden auf der Basis der Daten aus der vierten International „Civic and Citizenship Education“-Studie aus 14 europäischen Bildungssystemen $(N=46.500)$ zunächst die Effekte für das nationale und überstaatliche Vertrauen in Abhängigkeit von politischem Wissen und der Korruptionsfreiheit in den jeweiligen EU-Mitgliedsstaaten untersucht. Anschließend werden in vertiefenden Pfadanalysen auf der Basis der Daten aus NRW $(N=1451)$ die Beziehungen zwischen schulischen Lerngelegenheiten, Sozialbeziehungen, politischem Wissen und supranationalen Einstellungen in den Blick genommen. Dabei wird herausgearbeitet, dass insbesondere die zur Verfügung gestellten schulischen Lerngelegenheiten zu Europa und das politische Wissen die Einstellungen zur EU positiv beeinflussen.

In dem Artikel von Andreas Zick, Wilhelm Berghan und Nico Mokros wird die Frage diskutiert, ob es sich bei der Anfälligkeit für rechte und antidemokratische Orientierungen eigentlich um ein ,Jugendproblem“ handelt, wie es in politischen oder pädagogischen Programmen zur Demokratieförderung oft unterstellt wird. Deshalb wird von den Autoren auf der Basis der Daten einer aktuellen Bevölkerungsumfrage empirisch geprüft, ob es zwischen den menschenfeindlichen, rechtspopulistischen und rechtsextremen Orientierungen eigentlich Unterschiede bei den Altersgruppen der 18- bis 30-Jährigen, der 31- bis 60-Jährigen sowie den über 60-Jährigen gibt. Die Analyse zeigt, dass kleinere Gruppen aus der jüngeren Generation zwar einzelnen Einstellungsdimensionen wie dem Sozialdarwinismus und der Verharmlosung des Nationalsozialismus häufiger zustimmen als die Mitglieder aus den beiden älteren Generationen. In Bezug auf die ganze Breite menschenfeindlicher Einstellungen zeigen die Älteren jedoch höhere Zustimmungswerte. Abschließend werden auch unter Bezug auf die Ergebnisse anderer Studien die Bedingungsfaktoren für die skizzierten Einstellungsmuster diskutiert und einige Forschungsperspektiven für eine intergenerativ vergleichende politische Sozialisationsforschung angedeutet.

In dem Beitrag von Katharina Trittel und Julian Schenke wird auf der Grundlage der Daten einer qualitativen Studie untersucht, wie unterschiedlich Jugendliche und junge Erwachsene mit dem Protestphänomen „PEGIDA“ in ihren politischen Deutungsmustern umgehen. Dazu wird das Projekt in den aktuellen Forschungsstand und den theoretischen Diskurs um die neuen Konfliktlinien des Transnationalismus eingebettet. Anschließend wird das methodische Design der Studie beschrieben, und es wird auf der Basis der Ergebnisse der durchgeführten Gruppendiskussionen aufgezeigt, dass die Jugendlichen und jungen Erwachsenen in ihren Deutungsmustern zwar eine primäre Distanz zu PEGIDA zeigen, sich in ihren politischen Mentalitätsmustern jedoch zugleich Argumentationsfiguren herausarbeiten lassen, die an 
die Topoi dieser politischen Protestbewegung sekundär anschlussfähig sind. Ihre Deutungsmuster bewegen sich im Spannungsverhältnis zwischen einem programmatischen Bezug auf die multikulturelle Gesellschaft und abwertenden Ungleichwertigkeitsaussagen.

In dem Artikel von Mark Ellison, Garry Pollok und Robert Grimm werden ausgewählte Ergebnisse einer großen quantitativen, europäischen Jugendstudie vorgestellt, bei der über 16.000 Jugendliche im Alter zwischen 16 und 26 Jahren in 30 Regionen aus 14 verschiedenen Ländern in Europa zu ihrem Vertrauen und ihrer Zufriedenheit mit politischen Institutionen, zu ihrem politischen Interesse und Wissen sowie zu ihrer politischen Partizipationsbereitschaft befragt worden sind. Aus dem Kontext dieser Studie werden in dem Beitrag die Verbreitung unterschiedlicher politischer Partizipationsformen und deren Einflussfaktoren genauer untersucht. Dazu werden in einem ersten Schritt zunächst der internationale Forschungsstand sowie die Grundbegriffe zu diesem Themenfeld dargestellt. In einem zweiten Schritt werden die Ziele, Hypothesen und das Design der Studie skizziert. Anschließend werden in einem dritten Schritt ausgewählte deskriptive Resultate vorgestellt, die u. a. verdeutlichen, dass protestorientierte Aktionsformen der politischen Partizipation eher von Jugendlichen in südeuropäischen Ländern wie Spanien oder Griechenland favorisiert werden. Abschließend werden in einem vierten Schritt auf der Basis der Ergebnisse multipler logistischer Regressionen die demografischen, regionalen und sozialisatorischen Einflussfaktoren auf drei unterschiedliche Modelle politischer Partizipation in Gestalt traditioneller Aktionsformen wie etwa Unterschriftensammeln, protestorientierter Aktionsformen wie Demonstrationen oder Streiks und der klassischen politischen Wahlbeteiligung analysiert. Dabei wird herausgearbeitet, dass insbesondere die soziale Herkunft aus höheren sozialen Klassen und sowie die familiale Diskussion über politische Themen einen positiven Einfluss auf die Bereitschaft von Jugendlichen hat, sich an allen drei Partizipationsformen zu beteiligen.

Eine Sammelrezension von Cathleen Grunert und Katja Ludwig, in der aktuellere Neuerscheinungen aus dem Bereich der Jugendforschung zu dem Themenfeld Jugend und Politik vorgestellt werden, rundet den Schwerpunktteil ab.

Funding Open Access funding enabled and organized by Projekt DEAL.

Open Access Dieser Artikel wird unter der Creative Commons Namensnennung 4.0 International Lizenz veröffentlicht, welche die Nutzung, Vervielfältigung, Bearbeitung, Verbreitung und Wiedergabe in jeglichem Medium und Format erlaubt, sofern Sie den/die ursprünglichen Autor(en) und die Quelle ordnungsgemäß nennen, einen Link zur Creative Commons Lizenz beifügen und angeben, ob Änderungen vorgenommen wurden.

Die in diesem Artikel enthaltenen Bilder und sonstiges Drittmaterial unterliegen ebenfalls der genannten Creative Commons Lizenz, sofern sich aus der Abbildungslegende nichts anderes ergibt. Sofern das betreffende Material nicht unter der genannten Creative Commons Lizenz steht und die betreffende Handlung nicht nach gesetzlichen Vorschriften erlaubt ist, ist für die oben aufgeführten Weiterverwendungen des Materials die Einwilligung des jeweiligen Rechteinhabers einzuholen.

Weitere Details zur Lizenz entnehmen Sie bitte der Lizenzinformation auf http://creativecommons.org/ licenses/by/4.0/deed.de. 


\section{Literatur}

Abs, H. J., Hahn-Lautenberg, K., Deimel, D., \& Ziemer, J. (2017). Einleitung. In K. Hahn-Lautenberg \& H.J. Abs (Hrsg.), Das politische Mindset von 14-Jährigen. Ergebnisse der International Civic and Citizenship Education Study 2016 (S. 9-26). Münster: Waxmann.

Krüger, H.-H., \& Grunert, C. (2020). Historische Entwicklung und zukünftige Herausforderungen der Kindheits- und Jugendforschung. In H.-H. Krüger, C. Grunert \& K. Ludwig (Hrsg.), Handbuch Kindheits- und Jugendforschung (3. Aufl.) Wiesbaden: Springer VS. im Druck.

Schelsky, H. (1961). Die Skeptische Generation. Eine Soziologie der Jugend (1957). Düsseldorf: Eugen Diederich.

Shell Holding Deutschland (2019). Jugend 2019. Weinheim: Beltz. 